\title{
Review Article \\ Through-Wall Detection with LS-SVM under Unknown Wall Characteristics
}

\author{
Fangfang Wang, Yerong Zhang, and Huamei Zhang \\ School of Electronic Science and Engineering, Nanjing University of Posts and Telecommunications, Nanjing 210003, China \\ Correspondence should be addressed to Fangfang Wang; wangff@njupt.edu.cn
}

Received 27 October 2015; Revised 16 March 2016; Accepted 10 April 2016

Academic Editor: Francisco Falcone

Copyright (C) 2016 Fangfang Wang et al. This is an open access article distributed under the Creative Commons Attribution License, which permits unrestricted use, distribution, and reproduction in any medium, provided the original work is properly cited.

One of the main challenges in through-wall imaging (TWI) is the presence of the walls, whose returns tend to obscure the target behind the walls and must be considered and computed in the imaging procedure. In this paper, a two-step procedure for the through-wall detection is proposed. Firstly, an effective clutter mitigation method based on singular value decomposition (SVD) is used. It does not require knowledge of the background scene or rely on accurate modeling and estimation of wall parameters. Then, TWI problem is cast as a regression one and solved by means of least-squares support vector machine (LS-SVM). The complex scattering process due to the presence of the walls is automatically included in the nonlinear relationship between the feature vector extracted from the target scattered fields and the position of the target. The relationship is obtained through a training phase using LS-SVM. Simulated results show that the proposed approach is effective. We also analyze the impacts of training samples and signalto-noise ratio (SNR) on test detection accuracy. Simulated results reveal that the proposed LS-SVM based approach can provide comparative performances in terms of accuracy, convergence, robustness, and generalization in comparison with the support vector machine (SVM) based approach.

\section{Introduction}

Sensing through obstacles such as walls, doors, and other visually opaque materials using microwave signals is emerging as a powerful tool which supports a range of civilian and military applications [1]. Through-wall imaging (TWI) is a research field of increasing interest to detect and locate scatterers which are hidden beyond an opaque obstacle, such as a building wall [2].

The presence of the walls makes the TWI problem different and more challenging than free space imaging. Compensation of wall effects such as attenuation and dispersion must be accounted for in imaging procedure; otherwise it will lead to the degradation of imaging such as the shift of true positions and the smearing and blurring of the image $[3,4]$. This has been done by using some modified synthetic aperture radar (SAR) processing methods relying on an approximate model of the walls [5] or casting the original problem into an inverse scattering one governed by wave equations [6]. The problems of detecting the target behind the walls are usually formulated and solved as optimization problems which are solved using iterative minimization procedures. Iterative methods involve solving a well-behaved forward problem in a feedback loop. Thus, these methods are computationally expensive.

All of the imaging approaches mentioned above for TWI problem are based on physical modeling. However, the lack of required data and the expense of data acquisition can limit the practical application of physics-based models. In this situation, data-driven models are good alternative, or a complement, to physics-based models for TWI problem [7]. They are characterized by their ability to capture the underlying physics of the system simply by examination of the inputs and outputs of the system. Taking advantage of data-driven model, the TWI problem is reformulated as a regression one, where the data (i.e., scatter-fields measurement) and the unknowns (i.e., the position of the object) are related by means of an approximated function to be estimated through an offline data fitting process. In this way, the effects caused by the walls can be included in the mapping obtained after the training phase. Therefore, this technology is not sensitive to a priori knowledge on the walls or the room geometry. 
Recently, the support vector machine (SVM) has been successfully applied in solving regression problems and has shown excellent performances. SVM is developed on the basis of statistic learning theory and exhibits good generalization capabilities thanks to an intrinsic tradeoff between fitting of the training data and model complexity $[8,9]$. Nonlinear SVM provides robust solutions by mapping the input space into a higher dimensional feature space using kernel functions. However, the SVM is obtained using quadratic programming methods, which can be time-consuming and difficult to implement [10]. Least-squares support vector machine (LS-SVM) is a reformulation of the standard SVM [11]. LS-SVM adopts equality constraints instead of the inequality constraints implemented in SVM and a quadratic error term to obtain a linear set of equations in a dual space. LS-SVM performs well for its simple calculation, faster convergence, and higher precision than traditional SVM [12].

The above imaging methods are based on the assumption that the scattered fields from the target have been already extracted from the total fields. Generally, the strong clutter induced by the walls and crosstalk between antennas often obscure the target electromagnetic (EM) returns. Therefore, a clutter mitigation technique is also required before applying imaging algorithms.

Recently, clutter mitigation based on statistical techniques such as singular value decomposition (SVD) has been introduced in TWI applications successfully $[13,14]$. The clutter mitigation technique based on SVD does not require a priori information wall parameters. The target signal is reconstructed using singular values and vectors corresponding to the target based on the different property compared to the clutter.

Inspired by the advantage of LS-SVM and SVD algorithms, we introduce in this paper a two-stage procedure for detection of the target behind the walls under wall parameters ambiguities. Firstly, the total received data is dealt with to mitigate the clutter including the wall reflection and crosstalk between antennas using SVD. Then, some features are extracted from the target scattered fields and a correspondence between the feature vector and target information such as positions is established by LS-SVM using the training data. For a new detection task, the target positions are regressed by the correspondence.

The remainder of this paper is organized as follows. In Section 2, we present the through-wall detection problem briefly and SVD is introduced to mitigate the clutter from the B-scan radar data. Then, we recast the problem as a regression one. In Section 3, LS-SVM is introduced and used for solving the regression problem. In Section 4, numerical results for synthetic data are shown, and the performances of the proposed through-wall detection technique are assessed. Finally, some conclusions and final remarks are made.

\section{Problem Description}

TWI scenario in this paper is referring to a three-layer media model as depicted in Figure 1. The first and third layers are assumed to be the free space with dielectric permittivity and

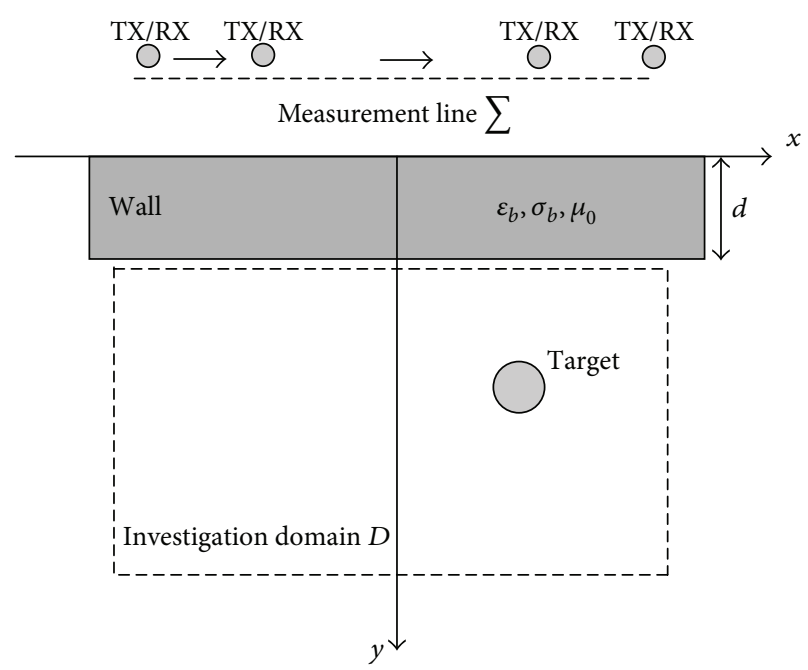

FIGURE 1: Geometry of the scattering problem.

magnetic permeability denoted as $\varepsilon_{0}$ and $\mu_{0}$, respectively. The second layer is the nonmagnetic wall with thickness $d$ and permittivity $\varepsilon_{b}$ and conductivity is $\sigma_{b}$. The target is located in the investigation domain $D$. The antenna works in a monostatic mode; the reflected fields are collected at the same positions as the transmitting antenna, while the latter moves over a set of $N$ different uniform positions in order to synthesize a measurement line $\sum$.

When the wave propagates through the wall, it will reflect at the air-to-wall and wall-to-air interfaces causing multiple reverberations within the wall [15]. Returns also involve multiple bounces between the wall and target, so the whole receiving signal is a nonlinear system. This gives rise to ghost targets and hence increases probability of false alarm. It is another challenge in through-wall imaging and is not considered in this work. A common way to model a throughwall scene is to consider it as a linear system, so the received echo represents the total contributions of wall reflection and target scattering fields and noise written as

$$
e_{n}(t)=e_{n a}(t)+e_{n w}(t)+e_{n t}(t),
$$

where $e_{n a}(t)$ accounts for the antenna crosstalk and $e_{n w}(t)$ is the wall reflection collectively called the clutter. $e_{n t}(t)$ is the fields scattered from the target behind the walls. Upon discretizing time, data can be arranged in a $M \times N$ B-scan matrix as

$$
\mathbf{B}=\left(\mathbf{b}_{1}, \mathbf{b}_{2}, \ldots, \mathbf{b}_{N}\right),
$$

where $\mathbf{b}_{j}=\left(y_{j 1}, y_{j 2}, \ldots, y_{j M}\right)^{T}$ represents the A-scan data collected at the $j$ th measurement position. $N$ and $M$ are the number of sensor positions and time samples, respectively.

In TWI scenario, the signal scattered from the target is overwhelmed by the clutter. The clutter mitigation is becoming the key to detecting the target without any wall parameters information. Therefore, the first step is to extract 
the target scattered fields $e_{n t}(t)$ from the total fields $e_{n}(t)$. The $\mathrm{SVD}$ of $\mathbf{B}$ is given as

$$
\mathbf{B}=\mathbf{U S V}^{H},
$$

where $\mathbf{U}=\left[\mathbf{u}_{1}, \mathbf{u}_{2}, \ldots, \mathbf{u}_{M}\right]$ and $\mathbf{V}=\left[\mathbf{v}_{1}, \mathbf{v}_{2}, \ldots, \mathbf{v}_{N}\right]$ are unitary matrices, respectively. $\mathbf{u}_{i}=\left[u_{1 i}, u_{2 i}, \ldots, u_{M i}\right]^{H}$ and $\mathbf{v}_{i}=\left[v_{1 i}, v_{2 i}, \ldots, v_{N i}\right]^{H}$ are the eigenvectors of $\mathbf{B B}^{H}$ and $\mathbf{B}^{H} \mathbf{B}$. $\mathbf{S}=\operatorname{diag}\left(\sigma_{1}, \sigma_{2}, \ldots, \sigma_{r}\right)$ and $\sigma$ are the singular values with the order $\sigma_{1} \geq \sigma_{2} \geq \cdots \geq \sigma_{r} \geq 0$. Therefore, $\mathbf{B}$ can be expressed as a linear combination of all eigencomponents:

$$
\mathbf{B}=\sum_{n=1}^{r} \mathbf{E}_{n}=\sigma_{1} \mathbf{u}_{1} \mathbf{v}_{1}{ }^{H}+\cdots+\sigma_{r} \mathbf{u}_{r} \mathbf{v}_{r}{ }^{H}
$$

where $\mathbf{E}_{n}$ stands for the $n$th eigencomponent. $\mathbf{B}$ can be decomposed into two subspaces, target $\mathbf{B}_{C}$ and clutter $\mathbf{B}_{T}$, respectively:

$$
\mathbf{B}=\mathbf{B}_{C}+\mathbf{B}_{T}=\sum_{n=1}^{p} \sigma_{n} \mathbf{u}_{n} \mathbf{v}_{n}{ }^{H}+\sum_{n=p+1}^{r} \sigma_{n} \mathbf{u}_{n} \mathbf{v}_{n}{ }^{H} .
$$

$p$ is the boundary of the two subspaces.

In monostatic radar scheme, the amplitude and phase of the clutter at each antenna location across the synthetic aperture are the same. As a result, the clutter measurement vectors corresponding to various antenna locations are linearly dependent leading to the rank of the clutter data matrix being one. When applying SVD to the data matrix, the clutter, due to its strength relative to the target, resides in the first singular vector corresponding to the maximal singular value. Therefore, after removing the singular vector corresponding to the maximal singular value, the residual singular vectors are used to construct the target subspace. The target subspace can be written as

$$
\mathbf{B}_{T}=\sum_{n=2}^{r} \sigma_{n} \mathbf{u}_{n} \mathbf{v}_{n}{ }^{H} .
$$

TWI aims at retrieving the location, the shape, and the dielectric property of the target from the measured fields when illuminated by known incident fields. The target scattered fields can be thought of as a function of their parameters. Therefore, TWI problem can be considered as a regression one, which involves estimating or approximating the underlying relationship from detected signal data set. Generally, one target is centered at $\left(p_{-} x, p_{-} y\right)$ with a diameter of $\rho_{t}$, and the conductivity and dielectric permittivity of it are characterized by $\sigma_{t}, \varepsilon_{t}$, respectively. $\mathbf{x}$ is the feature vector extracted from the target scattered fields $\mathbf{B}_{T}$ such as a set of amplitude at each observation location, and $\mathbf{y}=$ $\left(p_{-} x, p_{-} y, \rho_{t}, \varepsilon_{t}, \sigma_{t}\right)$ represents the target parameters. Once the mapping from $\mathbf{x}$ to $\mathbf{y}$ is established, we can predict the vector $\mathbf{y}$ from the data vector $\mathbf{x}$.

\section{LS-SVM for Function Regression}

The training dataset $\left\{\left(\mathbf{x}_{1}, y_{1}\right), \ldots,\left(\mathbf{x}_{N}, y_{N}\right)\right\} \subset R^{N} \times R$ is given, where $R^{N}$ denotes the space of the input patterns; $\mathbf{x}_{i}$ represents the scattered fields due to the target, and $y_{i}$ represents the position of the target such as $x$-coordinate or $y$-coordinate (i.e., $y$ is an element of the vector $\mathbf{y}$ ) for the through-wall detection. The LS-SVM model for nonlinear function regression is represented in feature space as

$$
y(\mathbf{x})=\mathbf{w}^{T} \varphi(\mathbf{x})+b .
$$

Here, the nonlinear function $\varphi(\cdot): R^{N} \rightarrow R^{N_{k}}$ maps the input space to a higher-dimension feature space. $b$ is a bias term and $\mathbf{w} \in R^{N}$ is the weight vector. The optimization problem consists in minimizing

$$
J(\mathbf{w}, \mathbf{e})=\frac{1}{2} \mathbf{w}^{T} \mathbf{w}+\gamma \frac{1}{2} \sum_{i=1}^{N} e_{i}^{2}
$$

subject to the equality constraints

$$
y_{i}=\mathbf{w}^{T} \varphi\left(\mathbf{x}_{i}\right)+b+e_{i} \quad i=1, \ldots, N,
$$

where the fitting error is denoted by $e_{i}$. The hyperparameter $\gamma$ controls the tradeoff between the smoothness of the function $y$ and the accuracy of the fitting. The solution is obtained after constructing the Lagrangian

$$
\begin{aligned}
L(\mathbf{w}, b, \mathbf{e}, \boldsymbol{\alpha})= & J(\mathbf{w}, \mathbf{e}) \\
& -\sum_{i=1}^{N} \alpha_{i}\left\{\mathbf{w}^{T} \varphi\left(\mathbf{x}_{i}\right)+b+e_{i}-y_{i}\right\},
\end{aligned}
$$

where $\alpha_{i}$ are Lagrangian multipliers. The optimality of upper function is as the following sets of linear equations:

$$
\begin{aligned}
& \frac{\partial L}{\partial \mathbf{w}}=0 \longrightarrow \mathbf{w}=\sum_{i=1}^{N} \alpha_{i} \varphi\left(\mathbf{x}_{i}\right) \\
& \frac{\partial L}{\partial b}=0 \longrightarrow \sum_{i=1}^{N} \alpha_{i}=0 \\
& \frac{\partial L}{\partial e_{i}}=0 \longrightarrow \alpha_{i}=\gamma e_{i} \\
& \frac{\partial L}{\partial \alpha_{i}}=0 \longrightarrow \mathbf{w}^{T} \varphi\left(\mathbf{x}_{i}\right)+b+e_{i}-y_{i}=0 .
\end{aligned}
$$

After eliminating variables $\mathbf{w}$ and $\mathbf{e}$, we get matrix equations:

$$
\left[\begin{array}{cc}
0 & \mathbf{1}^{T} \\
\mathbf{1} & \boldsymbol{\Omega}+\gamma^{-1} \mathbf{I}
\end{array}\right]\left[\begin{array}{l}
b \\
\boldsymbol{\alpha}
\end{array}\right]=\left[\begin{array}{l}
0 \\
\mathbf{y}
\end{array}\right],
$$

where $\mathbf{y}=\left[y_{1} ; \ldots ; y_{N}\right], \mathbf{1}=[1 ; \ldots ; 1], \boldsymbol{\alpha}=\left[\alpha_{1} ; \ldots ; \alpha_{N}\right]$, and $\boldsymbol{\Omega}_{k l}=K\left(\mathbf{x}_{k}, \mathbf{x}_{l}\right)$ is the kernel matrix.

According to Mercer's condition, there is mapping $\varphi$ and kernel function

$$
K\left(\mathbf{x}_{k}, \mathbf{x}_{l}\right)=\varphi\left(\mathbf{x}_{k}\right)^{T} \varphi\left(\mathbf{x}_{l}\right) .
$$

The resulting LS-SVM model for nonlinear function regression becomes

$$
y(\mathbf{x})=\sum_{i=1}^{N} \alpha_{i} K\left(\mathbf{x}, \mathbf{x}_{i}\right)+b,
$$




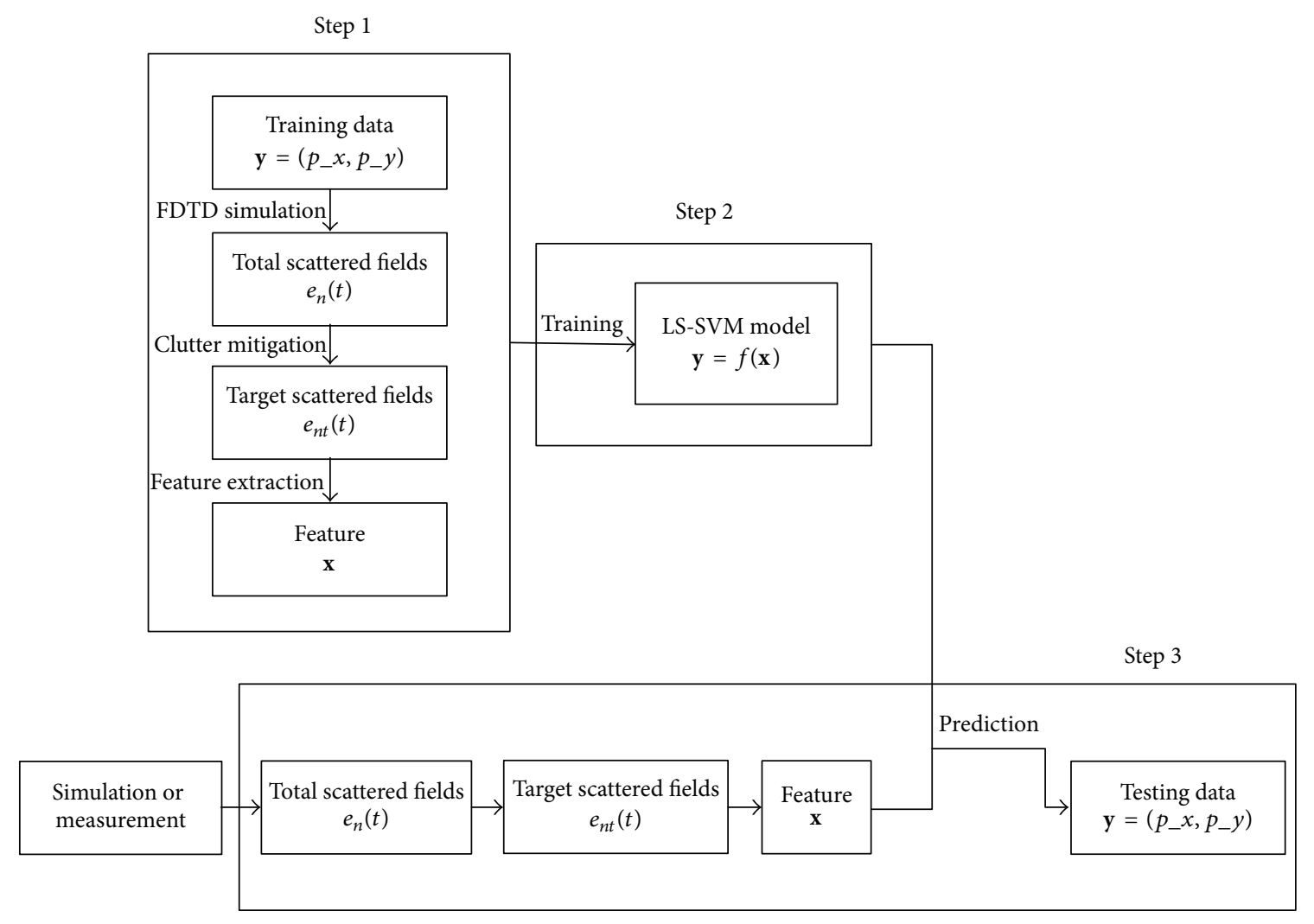

Figure 2: Procedure of the through-wall detection.

where $\alpha_{i}$ and $b$ are obtained by solving (12). The most usual kernel functions are linear, polynomial, and radial basis function (RBF). In this paper, RBF kernel is used:

$$
K\left(\mathbf{x}_{k}, \mathbf{x}_{l}\right)=\exp \left(-\frac{\left|\mathbf{x}_{k}-\mathbf{x}_{l}\right|^{2}}{2 \sigma^{2}}\right) .
$$

Here $\sigma$ is a constant defining the kernel width. The efficient performance of the LS-SVM model is determined by an optimal selection of the hyperparameters $\gamma$ and $\sigma$, which are tuned using cross-validation in the training phase. The whole procedure of the proposed method is shown in Figure 2.

\section{Results and Discussion}

In this section, the direct electromagnetic scattering problem, which is exploited to collect data, is formulated. Figure 3 shows the general scenario of an arbitrary dielectric scatterer residing in a simple room. The thickness of walls is $0.2 \mathrm{~m}$. The investigation domain is $D=[-1.08,1.08] \times[0.25,3.64] \mathrm{m}^{2}$. The conductivity $\sigma_{b}$ and relative permittivity $\varepsilon_{r}$ of the walls are set as a value of $0.003 \mathrm{~S} / \mathrm{m}$ and 2.75 , respectively.

The inside and outside regions of room (i.e., walls are excluded) are free space with dielectric permittivity and permeability denoted by $\varepsilon_{0}$ and $\mu_{0}$. The geometry of the room is discretized with the FDTD square cells of $1 \mathrm{~cm}$ length. The time resolution is $19.25 \times 10^{-12} \mathrm{~s}$. The antenna (TX/RX) transmits electromagnetic radiation and measures the radiation that is scattered back to the antenna. Then it moves and synthesizes a measurement aperture $L=2.4 \mathrm{~m}$. To model the EM illumination of the room and the object in it with an UWB short pulse, the transmitter dipole antenna is fed by a $4.5 \mathrm{~ns}$ Gaussian pulse modulated by a $0.5 \mathrm{GHz}$ sine wave.

When the observation points are $N=9$, the time domain responses are sampled and arranged into a B-scan matrix $\mathbf{B}$ as shown in Figure 4(a). It is shown that the target scattered fields are overwhelmed by the clutter signals. Using SVD, matrix $\mathbf{B}$ can be decomposed into several singular elements, and the singular values of the matrix are depicted in Figure 4(b). In monostatic radar model, a homogenous wall subspace can be assumed to be spanned by the first singular vector associated with the dominant singular value. Therefore, a SVD-based method is proposed to separate the clutter and the target subspace since the first singular element is to account for the clutter returns. After the clutter mitigation based on the proposed method, the target scattered fields are reconstructed as shown in Figure 5(a). The results based on background subtraction are depicted in Figure 5(b). From Figures 5(a) and 5(b), it shows that the proposed method is as effective as background subtraction in removing the clutter and revealing the target behind the wall, while it does not know a prior knowledge of the background scene. Figure 5(a) shows that there are also signals caused by mutual interaction between the wall and target after the clutter mitigation process. 


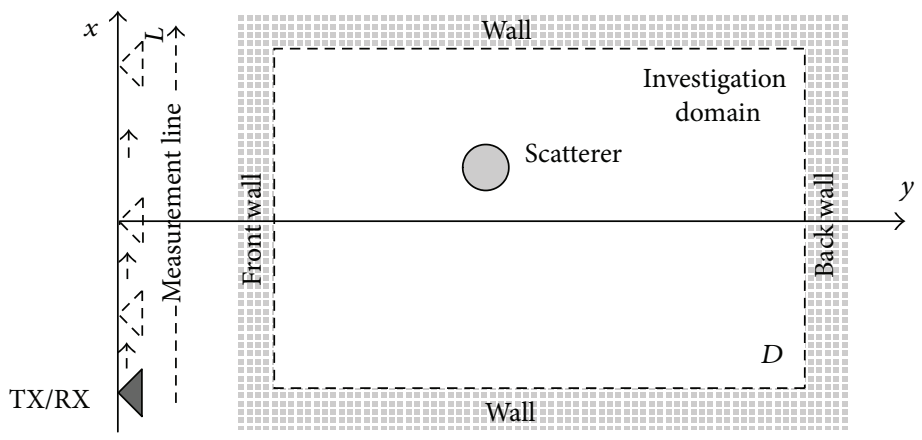

FIGURE 3: Schematic of through-wall geometry.

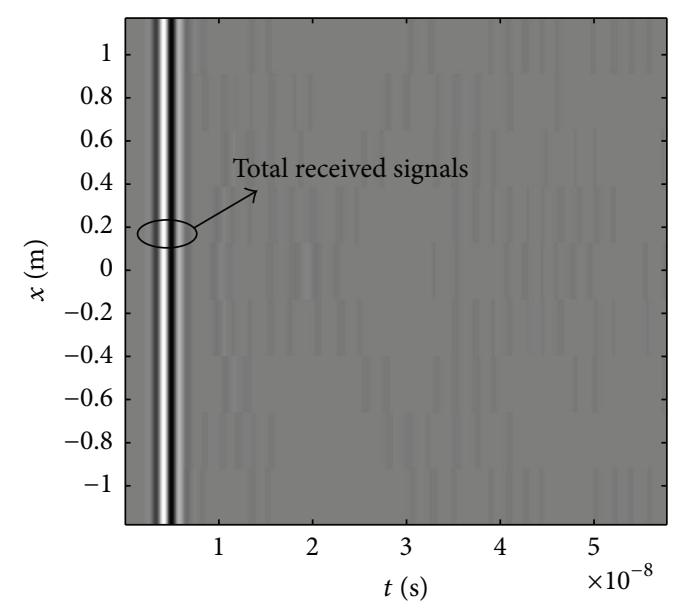

(a)

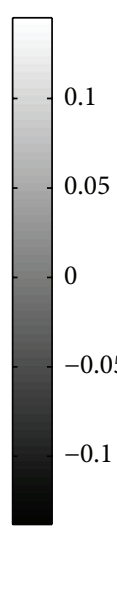

FIGURE 4: B-scan data for the total received fields and its singular values: (a) B-scan data and (b) singular values.

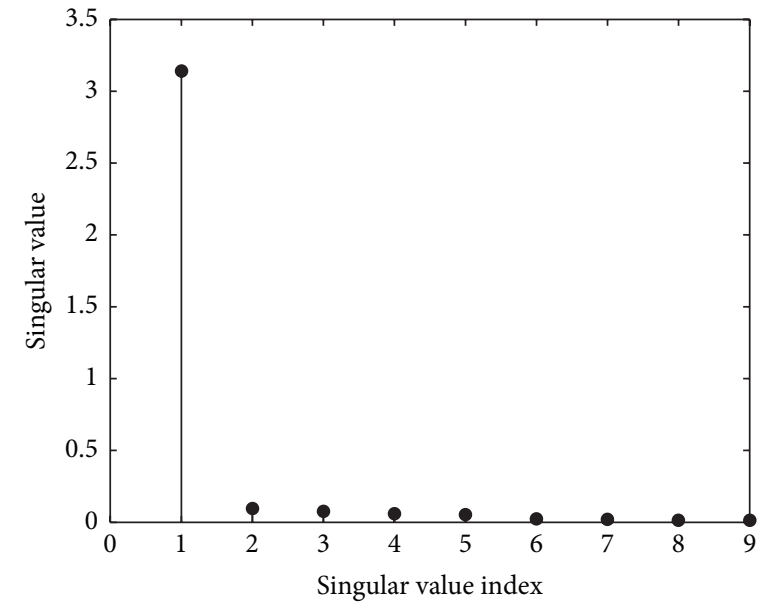

(b)

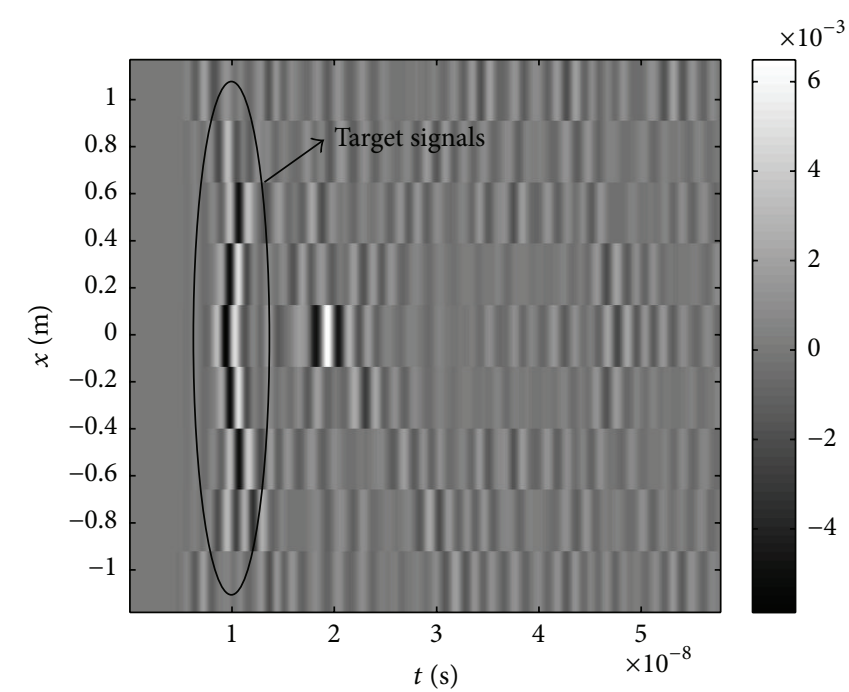

(a) 


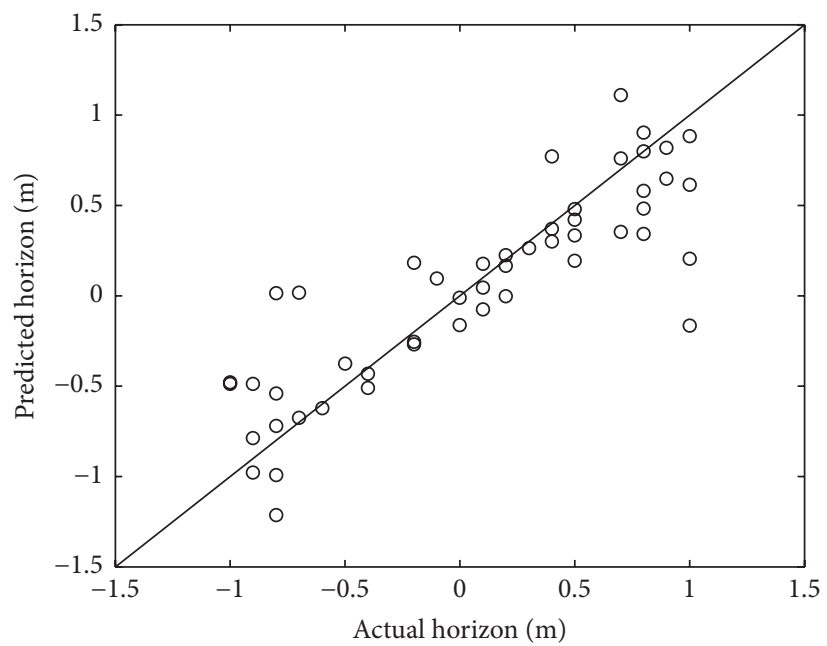

(a)

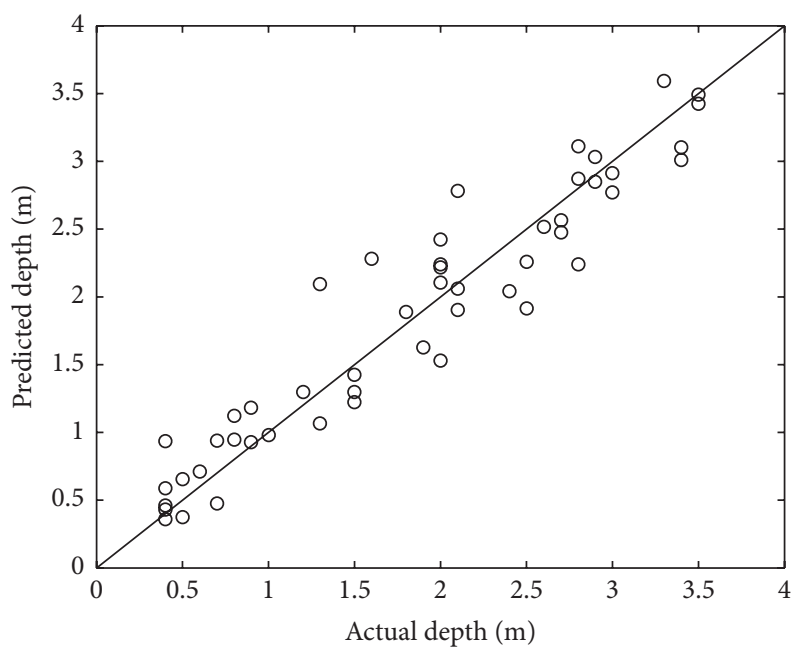

(b)

Figure 6: Predicted values of (a) horizon and (b) depth of the target center versus the actual ones.

Once obtaining the scattered fields from the target, some features can be extracted from these fields. In this paper, we consider singular values except for the first one, time of arrival, and amplitude of the scattered fields at individual observation location as the features. Ultimately, a vector containing these features is fed into LS-SVM predictor as an input and the position of the scatterer is the output.

The LS-SVM must be trained by exploiting some inputoutput patterns suitably chosen so as to give an exhaustive representation of the through-wall detection problem. To do this, the training dataset is achieved by repeated simulations with varying the position of the target as follows:

$$
\begin{aligned}
& p_{\perp} x_{n}=-1+n \Delta p_{-} x, \\
& p_{-} y_{n}=0.3+n \Delta p_{-} y, \\
& n=0,1, \ldots, N_{x}, \Delta p_{-} x=0.1 \mathrm{~m}, \\
& n=0, N_{y}, \Delta p_{-} y=0.1 \mathrm{~m},
\end{aligned}
$$

where $N_{x}=21, N_{y}=33$. The total 693 samples are divided into two sets, where 643 samples are selected as the training set to construct LS-SVM model and the remaining is selected as the testing set.

Since the assessment is focused on the target detection through a wall, the degree of matching between the actual and predicted position comprised of horizon and depth is firstly evaluated using the constructed LS-SVM model. The results are shown in Figure 6. The more the points are concentrated around the diagonal line, the better the prediction of the model. The results show that most of the points locate near the diagonal line.

We define the mean square error (MSE) as

$$
\text { MSE }=\frac{1}{N_{\text {test }}} \sum_{i=1}^{N_{\text {test }}}\left(p_{i}-q_{i}\right)^{2},
$$

where $N_{\text {test }}$ is the number of the samples in testing dataset and $p_{i}$ and $q_{i}$ are the $i$ th actual and predicted position of the target, respectively. Firstly, the MSE against the number of training samples using LS-SVM compared with the results obtained by SVM are illustrated in Figure 7.

The number of training samples changes from 200 to 643; it can be observed that the MSE decreases rapidly as a function of the number of training examples. Results in Figure 7 declare that it is necessary to increase the training samples to get higher detection accuracy if possible. However, the CPU time increases with the growth of the training set size. As a result, the tradeoff between two factors should be considered intensively.

The above-mentioned scene is for noiseless circumstance. In reality, it is important that the reconstruction algorithm is robust under noise contamination. To do this, after the feature vectors are generated, they are successively corrupted by an additive Gaussian noise with mean value equal to zero and variance fixed according to the desired signal-to-noise ratio (SNR). The MSE as a function of SNR is displayed in Figure 8 . It can be shown that the added noise almost has no effect on through-wall detection when using SVM model. However, the predicted error decreases rapidly as a function of the SNR when using LS-SVM model.

\section{Conclusion}

In this paper, we have presented a LS-SVM based method for target detection in through-wall imaging. SVD is firstly applied to remove or at least significantly suppress the clutter. Then, a flexible mapping between the scattered fields and the position of the target behind the walls is build using LS-SVM model. Simulated results reveal that the proposed approach does not assume prior knowledge of the scene nor the wall EM characteristics. The proposed approach can achieve high detection accuracy and keep low computation complexity. Moreover, the results confirm that the LS-SVM predictor can closely match the SVM predictor performances in terms of accuracy, convergence, robustness, and generalization. 


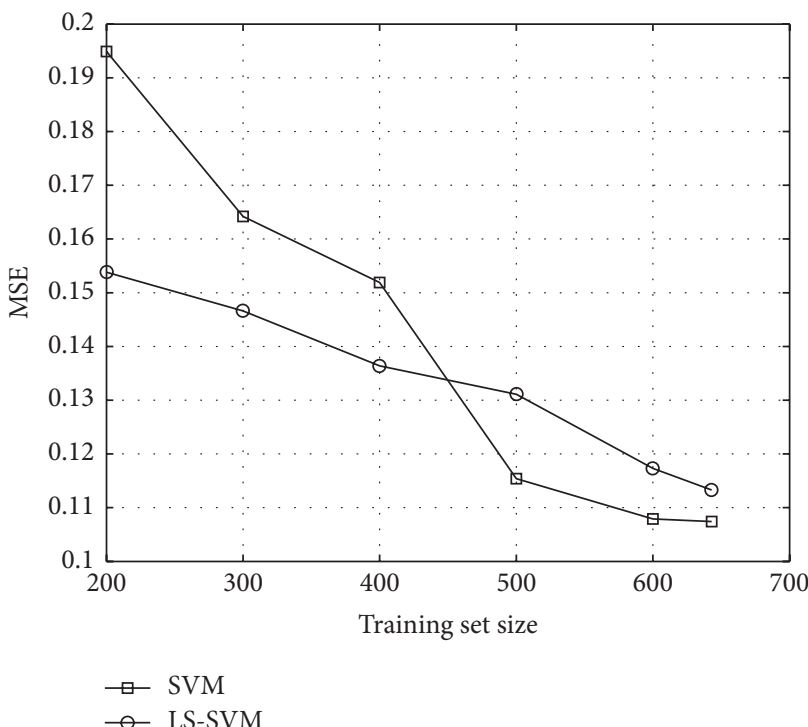

(a)

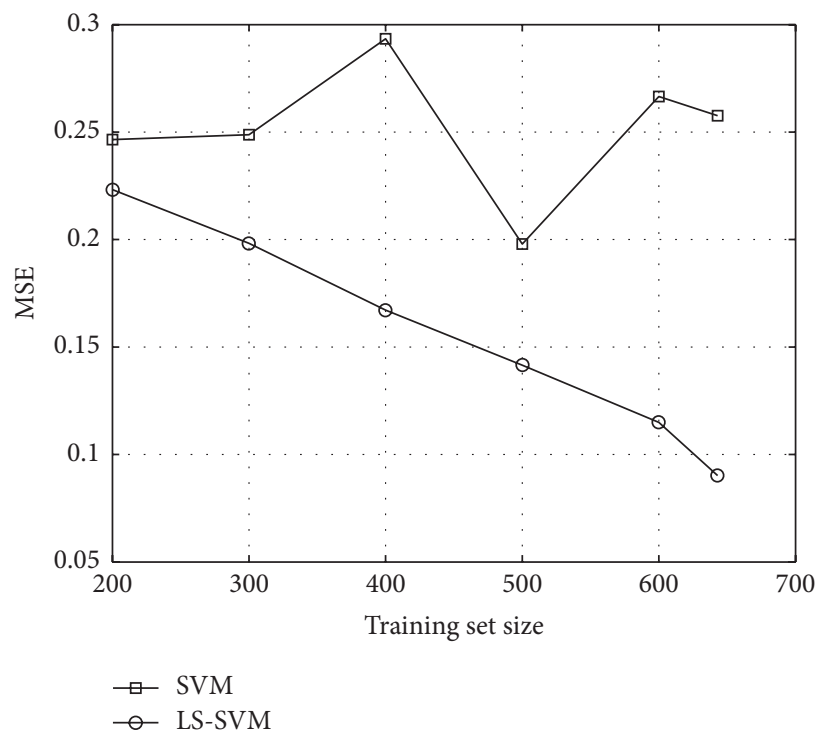

(b)

FIGURE 7: Impact of the number of training samples on MSE for (a) horizon and (b) depth of the target center.

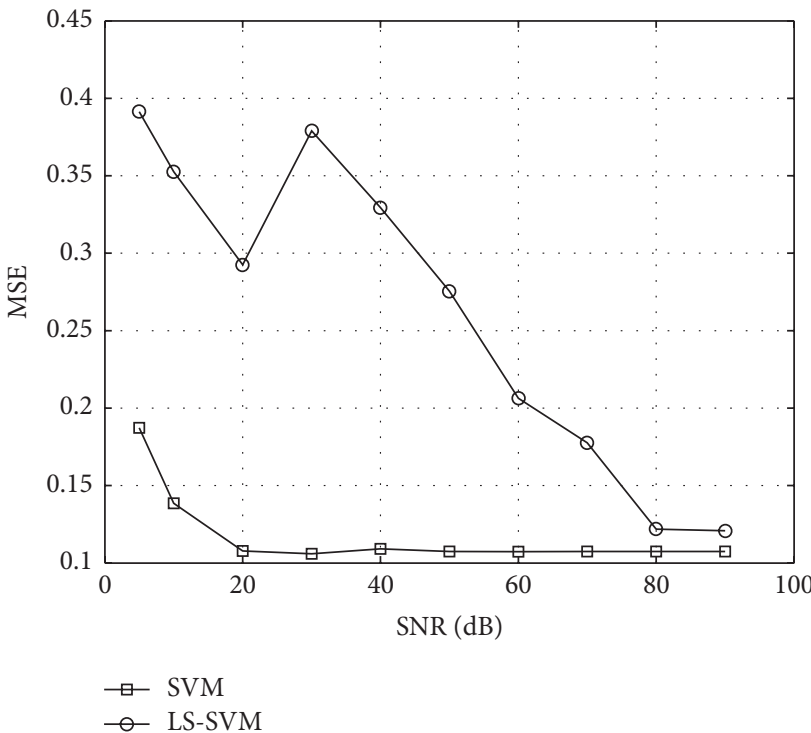

(a)

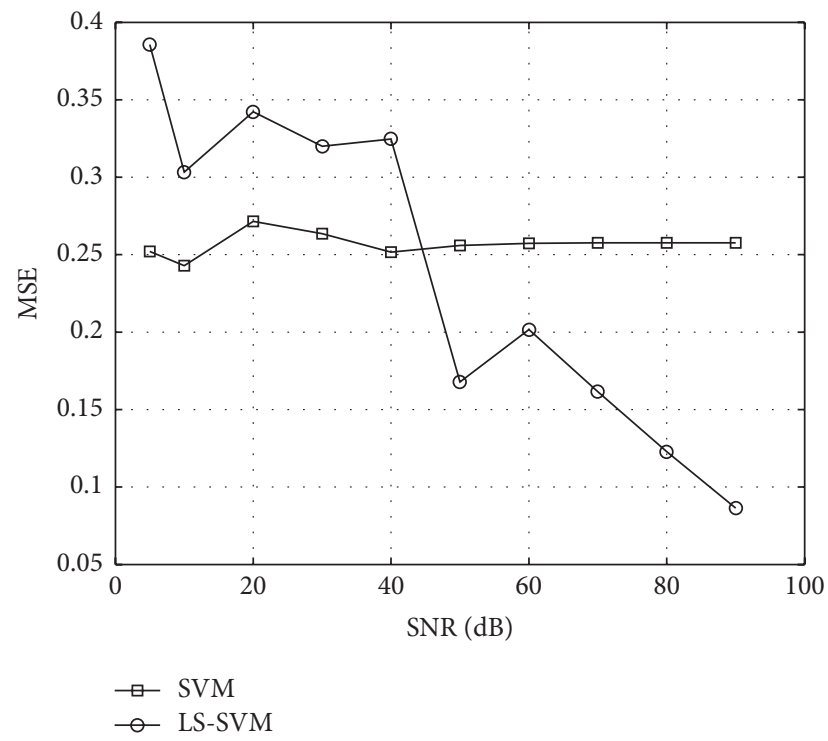

(b)

FIGURE 8: MSE as a function of SNR for (a) horizon and (b) depth of the target center.

\section{Competing Interests}

The authors declare that they have no competing interests.

\section{Acknowledgments}

This work is supported by the National Natural Science Foundation of China (Grant no. 61401225), Jiangsu Provincial National Science Foundation, China (Grant no. BK20140894), and Nanjing University of Posts and Telecommunications Foundation, China (Grant no. NY208037).

\section{References}

[1] M. G. Amin, Through-the-Wall Radar Imaging, CRC Press, New York, NY, USA, 2011.

[2] T. S. Ralston, G. L. Charvat, and J. E. Peabody, "Realtime through-wall imaging using an ultrawideband multipleinput multiple-output (MIMO) phased array radar system," in Proceedings of the 4th IEEE International Symposium on Phased Array Systems and Technology (ARRAY '10), pp. 551-558, Waltham, Mass, USA, October 2010.

[3] R. Solimene, F. Soldovieri, G. Prisco, and R. Pierri, "Threedimensional through-wall imaging under ambiguous wall 
parameters," IEEE Transactions on Geoscience and Remote Sensing, vol. 47, no. 5, pp. 1310-1317, 2009.

[4] R. Solimene, R. Di Napoli, F. Soldovieri, and R. Pierri, “TWI for an unknown symmetric lossless wall," IEEE Transactions on Geoscience and Remote Sensing, vol. 49, no. 8, pp. 2876-2886, 2011.

[5] F. Ahmad, M. G. Amin, and S. A. Kassam, "Synthetic aperture beamformer for imaging through a dielectric wall," IEEE Transactions on Aerospace and Electronic Systems, vol. 41, no. 1, pp. 271-283, 2005.

[6] F. Soldovieri and R. Solimene, "Through-wall imaging via a linear inverse scattering algorithm," IEEE Geoscience and Remote Sensing Letters, vol. 4, no. 4, pp. 513-517, 2007.

[7] Y. Kim and H. Ling, "Through-wall human tracking with multiple doppler sensors using an artificial neural network," IEEE Transactions on Antennas and Propagation, vol. 57, no. 7, pp. 2116-2122, 2009.

[8] A. J. Smola and B. Scholkopf, "A tutorial on support vector regression," Statistics and Computing, vol. 14, no. 3, pp. 199-222, 2004.

[9] F.-F. Wang and Y.-R. Zhang, "The support vector machine for dielectric target detection through a wall," Progress in Electromagnetics Research Letters, vol. 23, pp. 119-128, 2011.

[10] T. Hacib, Y. Le Bihan, M. K. Smail, M. R. Mekideche, O. Meyer, and L. Pichon, "Microwave characterization using ridge polynomial neural networks and least-square support vector machines," IEEE Transactions on Magnetics, vol. 47, no. 5, pp. 990-993, 2011.

[11] T. Van Gestel, J. A. K. Suykens, D.-E. Baestaens et al., "Financial time series prediction using least squares support vector machines within the evidence framework," IEEE Transactions on Neural Networks, vol. 12, no. 4, pp. 809-821, 2001.

[12] G. L. Sun and W. Guo, "Robust mobile geo-location algorithm based on LS-SVM," IEEE Transactions on Vehicular Technology, vol. 54, no. 3, pp. 1037-1041, 2005.

[13] E. Lagunas, M. G. Amin, F. Ahmad, and M. Nájar, "Joint wall mitigation and compressive sensing for indoor image reconstruction," IEEE Transactions on Geoscience and Remote Sensing, vol. 51, no. 2, pp. 891-906, 2013.

[14] F. H. C. Tivive, A. Bouzerdoum, and M. G. Amin, "A subspace projection approach for wall clutter mitigation in throughthe-wall radar imaging," IEEE Transactions on Geoscience and Remote Sensing, vol. 53, no. 4, pp. 2108-2122, 2015.

[15] A. T. Abdalla, A. H. Muqaibel, and S. Al-Dharrab, "Aspect dependent multipath ghost suppression in twri under compressive sensing framework," in Proceedings of the International Conference on Communications, Signal Processing, and their Applications (ICCSPA '15), pp. 1-6, Sharjah, UAE, February 2015. 


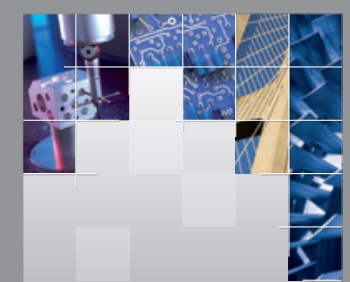

\section{Enfincering}
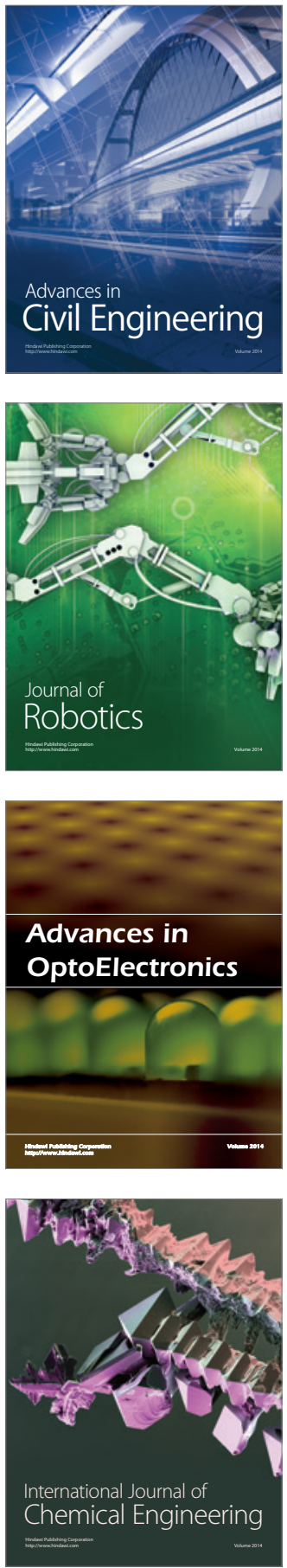

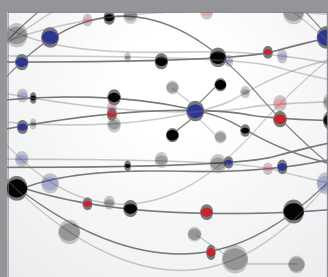

The Scientific World Journal

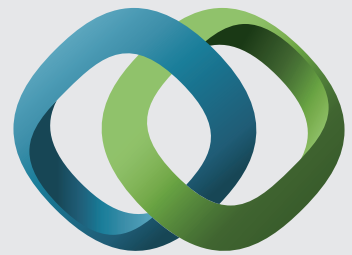

\section{Hindawi}

Submit your manuscripts at

http://www.hindawi.com
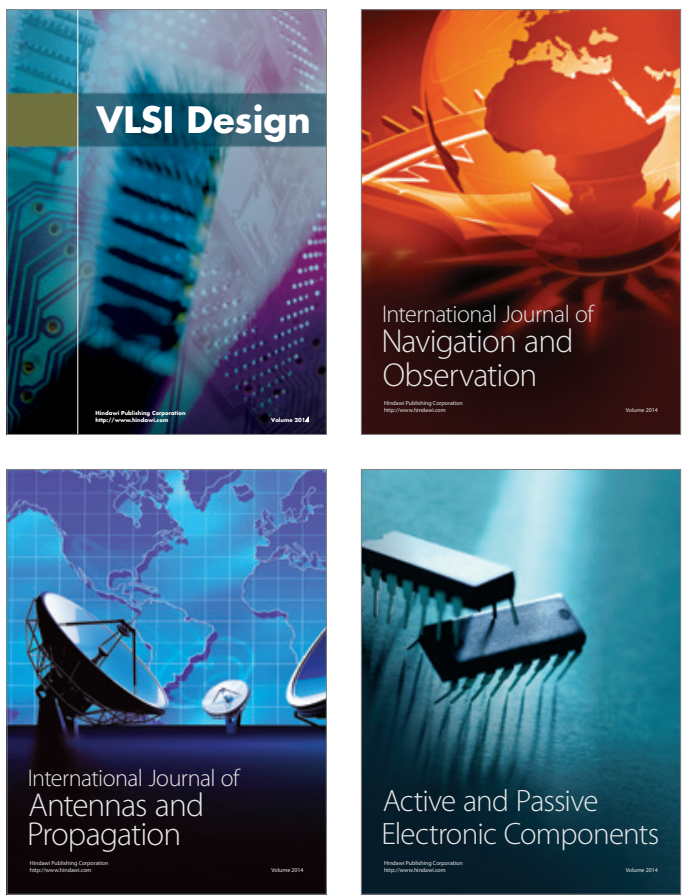
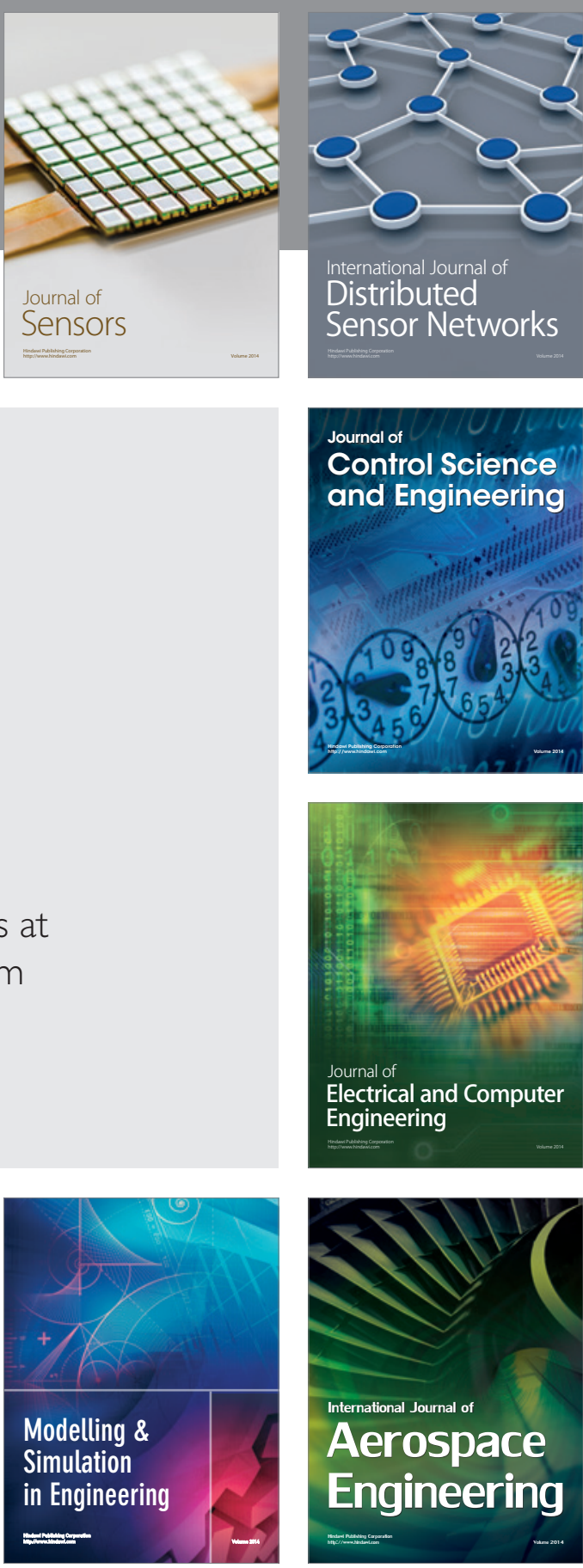

International Journal of

Distributed

Sensor Networks

Journal of

Control Science

and Engineering
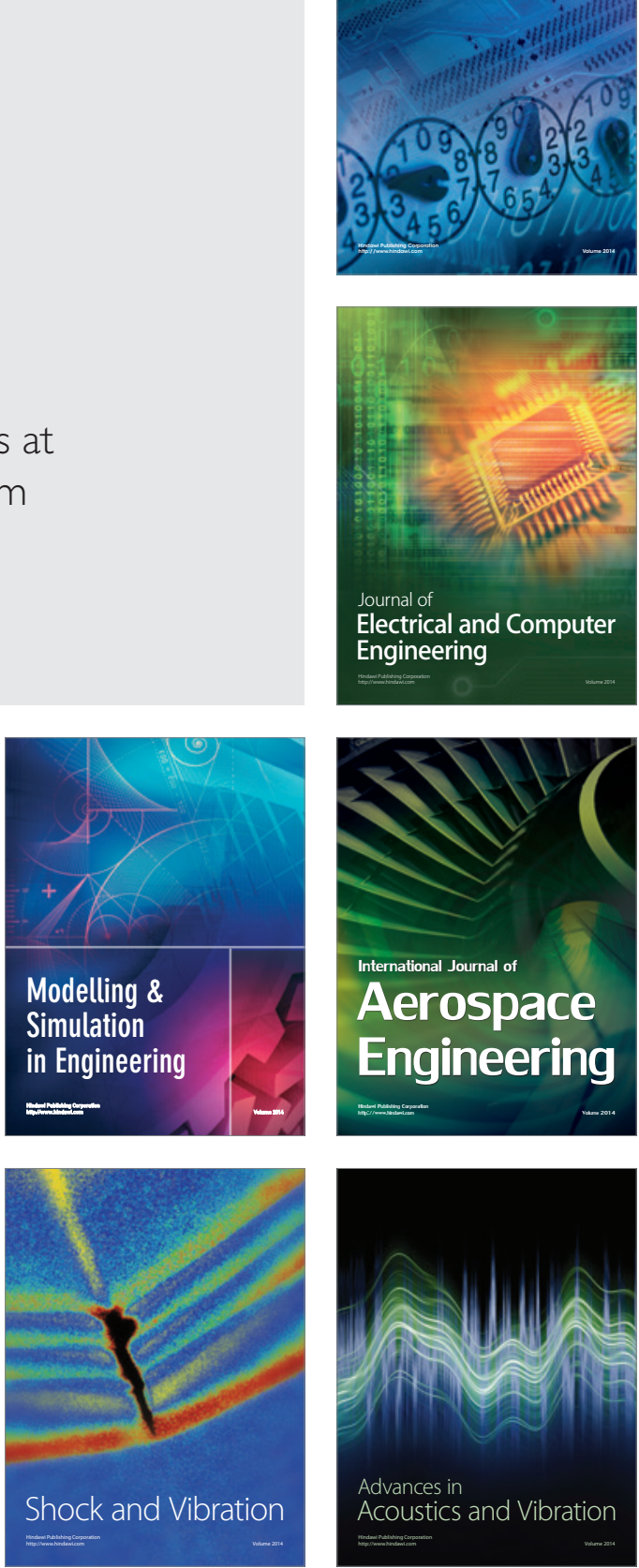